\title{
A FIVE-COLOR THEOREM FOR GRAPHS ON SURFACES
}

\author{
JOAN P. HUTCHINSON
}

\begin{abstract}
We prove that if a graph embeds on a surface with all edges suitably short, then the vertices of the graph can be five-colored. The motivation is that a graph embedded with short edges is locally a planar graph and hence should not require many more than four colors.
\end{abstract}

Introduction. It is well known $[4,15]$ that a graph embedded on a surface of genus $k \geqslant 0$, the sphere with $k$ handles, can always be $H(k)$-colored, where $H(k)=$ $[(7+\sqrt{48 k+1}) / 2] ; H(k)$ is called the Heawood number of the surface. A variety of properties are known which ensure that an embedded graph needs significantly fewer than $H(k)$ colors, for example, large girth [12, 13], few triangles (for graphs on the sphere or torus and, more generally, on surfaces of nonnegative Euler characteristic) $[9,11]$ and Eulerian properties of the (topological) dual graph [10].

On the other hand, one can look for properties which ensure that an embedded graph is locally a planar graph and hence needs not many more than four colors. In this spirit, Mycielski [14] has asked whether for every surface $S$ there is an $\varepsilon>0$ such that a graph embedded on $S$ with edges of length less than $\varepsilon$ can be five-colored. We restate Mycielski's question in terms of an explicit metric and then answer it in the affirmative for all surfaces. Work of Albertson and Stromquist [3] has already settled the case for the torus $(k=1)$, and we use many of their techniques in our proof. Also in [3] examples due to J. P. Ballantine and S. Fisk are given which show that no similar result for four-colorability is possible for any surface of positive genus.

A surface of genus $k \geqslant 1$ can be represented as a $4 k$-sided polygon with pairs of sides identified $[7,16,18$ ]. If a graph is embedded on a surface of genus $k \geqslant 1$, we obtain a representation $G_{k}$ of $G$ in and on the boundary of the $4 k$-gon. Without loss of generality we take the polygon to be a regular $4 k$-gon with sides of unit length; we call this the standard $4 k$-gon, $P_{k}$. Each edge of $G$ is represented in $G_{k}$ by one or more arcs in $P_{k}$ (if an edge crosses the boundary of $P_{k}$, it is divided into pieces). As explained in [5, p. 16], we may assume that each arc of $G_{k}$ is a polygonal arc; then by the length of an edge of an embedded graph $G$ we mean the sum of the lengths of its polygonal arcs in the representation $G_{k}$. Thus length is always defined in terms of a fixed representation of the graph on a standard polygon.

Our main result is the following.

Received by the editors March 30, 1983. Presented at the 806th meeting of the AMS, October 29, 1983. 1980 Mathematics Subject Classification. Primary 05C.15, 05C10.

Key words and phrases. Vertex coloring, embedded graph. 
THEOREM 1. Suppose $G$ has a 2-cell embedding on a surface of genus $k \geqslant 1$ and suppose $G$ has a representation $G_{k}$ on the standard $4 k$-gon such that every edge of $G$ has length less than $\varepsilon=1 / 5$. Then $G$ can be five-colored.

For the torus Albertson and Stromquist [3] have shown that a graph embedded with all noncontractible cycles of length at least 8 can be five-colored. This implies Theorem 1 with $k=1$ and $\varepsilon=1 / 7$ since all noncontractible cycles on $P_{k}$ have (Euclidean) length at least 1 . Stromquist [17] has more recently shown that 5-colorability follows for toroidal graphs provided all noncontractible cycles have length at least 4 , giving Theorem 1 with $k=1$ and $\varepsilon=1 / 3$. They conjecture that for each $k \geqslant 1$, there is a bound $b_{k}$ such that every graph embedded on the surface of genus $k$ with all noncontractible cycles of length at least $b_{k}$ can be 5-colored. The value of the bound must depend on $k$ since there are 6-chromatic graphs of arbitrarily large girth [6]. A proof of their conjecture would give Theorem 1 with $\varepsilon=1 /\left(b_{k}-1\right)$; however, our result holds with a fixed value of $\varepsilon$ for all surfaces. On the other hand, their results and conjecture are more natural in that they use a metric intrinsic to the graph whereas Theorem 1 relies upon an external geometric one.

Although short edges (as defined here) imply that all noncontractible cycles are long, the converse does not hold; for all surfaces there are graphs with all noncontractible cycles long and with some long edges in every representation $G_{k}$. For example, the graph on the double torus in Figure 1 has all noncontractible cycles of length at least 6 ; it is a 4-colorable graph.

There is no loss of generality in our interpretation of Mycielski's question and in our definition of edge length for the following reasons. Suppose $S_{k}$, the sphere with

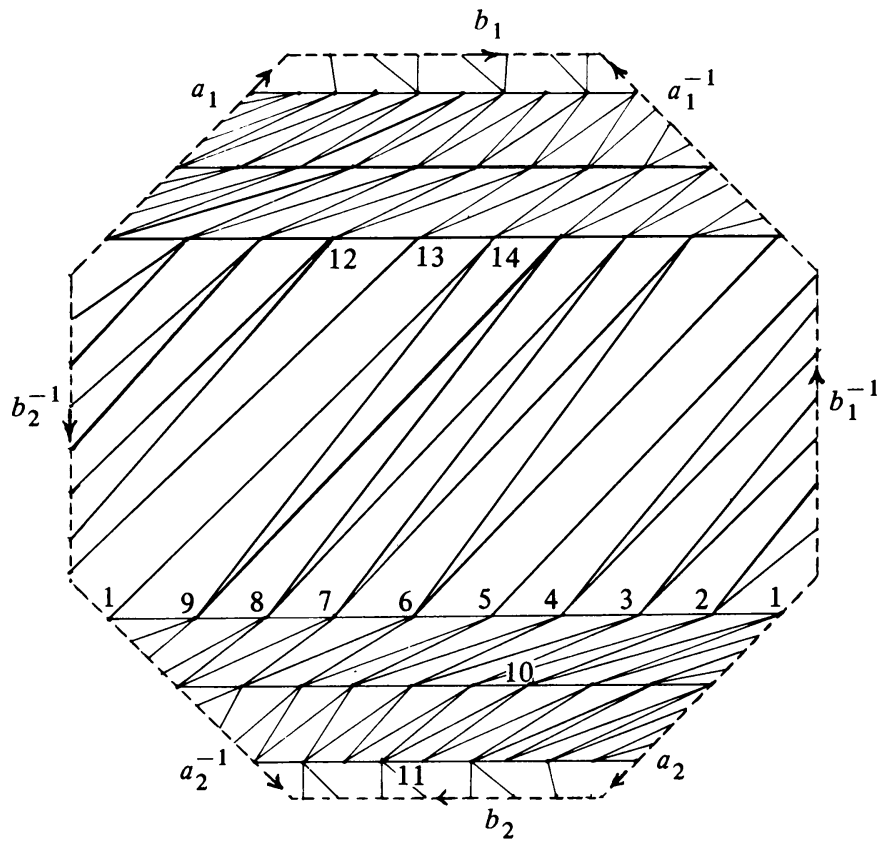

FIGURE 1 
$k$ handles, is taken to be some "nice" surface in $\mathbf{R}^{3}$ and that a graph $G$ embedded on $S_{k}$ has all edges rectifiable in $\mathbf{R}^{3}$. Then we may ask what bound on these edge lengths ensures that $G$ will be 5-colorable.

Suppose we define "nice" to mean that $S_{k}$ is a differentiable manifold ([16, §§2-3] gives a good introduction to this subject), and suppose we assume all edges of $G$ are piecewise differentiable curves on $S_{k}$ (as shown in [5], we lose no generality in this assumption). Then the length of each edge of $G$ can be determined by an integral; we denote the length of a piecewise differentiable curve $\gamma$ on $S_{k}$ by $\|\gamma\|_{1}$. Furthermore, there is a homeomorphism $f$ from $S_{k}$ to the standard $4 k$-gon $P_{k}$ in the plane, which is also differentiable. Then $f$ will map edges (or arbitrary piecewise differentiable curves $\gamma$ ) to piecewise differentiable curves in $P_{k}$; we denote the resulting lengths by $\|f(\gamma)\|_{2}$. Since $S_{k}$ and $P_{k}$ are compact, there are constants $c_{1}$ and $c_{2}$ such that $c_{1}\|\gamma\|_{1} \leqslant\|f(\gamma)\|_{2} \leqslant c_{2}\|\gamma\|_{1}$ for all piecewise differentiable curves $\gamma$ on $S_{k}$. Then we have the following consequence of Theorem 1.

COROllary 1. Suppose $G$ has a 2-cell embedding on a differentiable manifold of genus $k \geqslant 1$ and suppose every edge of $G$ is piecewise differentiable. Then if every edge of $G$ has length less than $1 /\left(5 c_{2}\right), G$ can be five-colored.

This follows by noting that the proof of Theorem 1 holds as well when the edges in the representation $G_{k}$ are piecewise differentiable.

Background in topological graph theory. We use basic graph theory terms as found in [18]. We consider only simple graphs and their 2-cell embeddings on surfaces, i.e. embeddings in which the interior of every face is a contractible (or null-homotopic) region. A 2-cell embedding implies that the graph is connected; there is no loss of generality in considering only 2-cell embeddings since any embedding of a connected graph can be transformed into a 2-cell inbedding by suitably cutting handles of the surface without affecting the graph embedding (see [18, p. 54]).

A cycle in a graph embedded on a surface is said to be contractible or noncontractible according as it is or is not homotopic to a point on the surface; we abbreviate the latter by calling it an nc-cycle. A cycle in an embedded graph is said to be null-homologous or non-null-homologous if it is an nc-cycle whose removal does or does not, respectively, disconnect the graph; we abbreviate the latter by calling it an nnh-cycle. In Figure 1 the graph shown on the double torus contains $C_{1}=\{1,9,14\}$, a contractible cycle, and $C_{2}=\{1,2,3,4,5,6,7,8,9\}$ or $C_{3}=\{1,10,11,12,13,14\}$, a non-null-homologous cycle. In Figure 2 a noncontractible and null-homologous cycle $C$ is marked in dashed lines; such cycles are present in the graph of Figure 1 but are long and not as illustrative. For the torus only, all nc-cycles are nnh, but for other surfaces the distinction is important (e.g. see [1]).

A cycle in a graph is said to be minimal if it contains no diagonal. If $C$ is a minimal nc-cycle, we arbitrarily give $C$ an orientation and define $R(C)$ and $L(C)$ to be the set of neighbors of $C$ which lie, respectively, to the right and to the left of $C$ on the surface as $C$ is traversed following the given orientation. $R(C)$ and $L(C)$ need not be disjoint. 


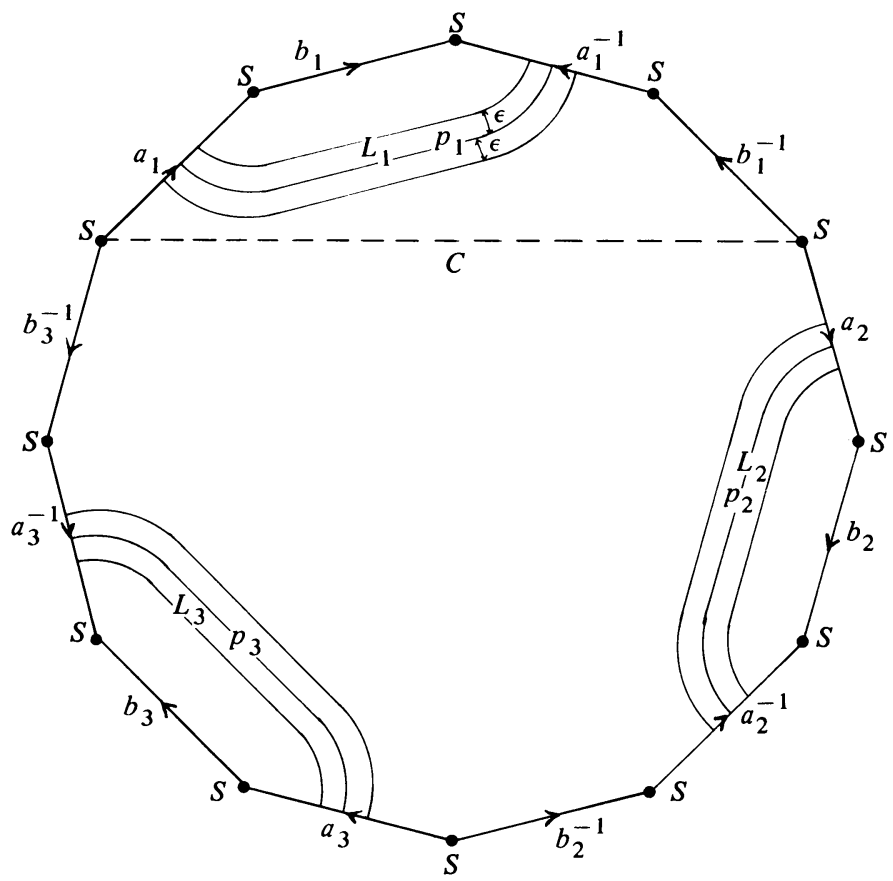

FIGURE 2

The final standard topological fact which we shall use is the following. Suppose $G$ has a 2-cell embedding on $S_{k}, k \geqslant 1$, and contains $k$ disjoint, pairwise nonhomotopic, nnh-cycles $C_{1}, C_{2}, \ldots, C_{k}$. Then deleting the vertices of $C_{1}, C_{2}, \ldots, C_{k}$ and their incident edges leaves a planar graph, since the elimination of $C_{1}, \ldots, C_{k}$ can be performed by cutting the surface along these cycles and sewing in $2 k$ discs, leaving a sphere [7, p. 63]. Further, if $C_{1}, \ldots, C_{k}$ are also minimal, we define $G\left(C_{1}, C_{2}, \ldots, C_{k}\right)$ to be the planar graph obtained by adding $2 k$ vertices to $G-\left\{C_{1}, \ldots, C_{k}\right\}$, two for each $i=1, \ldots, k$ : let $x_{R}^{i}$ be adjacent to each vertex of $R\left(C_{i}\right)$ and $x_{L}^{i}$ adjacent to each vertex of $L\left(C_{i}\right)$.

The next three lemmas are crucial to the proof of Theorem 1; the proofs of the first two can be found in [3]. Although in [3] these results are stated only for the torus, they were designed to be valid for all surfaces and hence yield Lemmas 1 and 2 as stated. Let a graph $G$ have a 2 -cell embedding on a surface of genus $k \geqslant 1$. The embedding is said to be orderly if $G$ is a triangulation, if every contractible 3-cycle is a face boundary, and if every contractible 4-cycle is either the first neighbor cycle of a vertex of degree 4 or the modulo 2 sum of two face boundaries with an edge in common.

Lemma 1. Let $G$ be a triangulation of a surface and $G_{0}$ the orderly triangulation obtained by deleting all vertices interior to a contractible 3- or 4-cycle and by subdividing any resulting quadrilateral. If $G_{0}$ can be 5-colored, then so can $G$.

LEMMA 2. Suppose $G$ has an orderly embedding on a surface and let $C$ be a minimal $n c$-cycle of length at least 4. Then within the induced (and embedded) subgraph of 
$C \cup R(C)$, there is a minimal nc-cycle which either has even length or contains a vertex of degree 4 (in $G$ ).

Such a cycle is called nice.

Suppose $C_{1}$ and $C_{2}$ are two disjoint, nonhomotopic, nnh-cycles in an embedded graph. We define $d\left(C_{1}, C_{2}\right)$ to be the length (number of edges) of the shortest path from a vertex of $C_{1}$ to one of $C_{2}$. We define $d\left(C_{i}, C_{i}\right), i=1,2$, to be the length of the shortest path joining two vertices of $C_{i}$, say $v$ and $w$ where possibly $v=w$, such that the path plus one segment of $C_{i}$ joining $v$ and $w$ is an nc-cycle, not homotopic to $C_{i}$. The resulting shortest nc-cycle is called $C_{i}^{*}$; the idea is that $C_{i}^{*}$ is the shortest cycle going around the same handle as $C_{i}$, but in a "different" direction. (For more details see $[2,3]$.)

We sketch the proof of the next result. It is only slightly different than that presented in [3], but it illustrates the coloring techniques involved.

Lemma 3. Suppose $G$ is an orderly triangulation of a surface of genus $k \geqslant 1$ and contains $k$ nice, nnh-cycles $C_{1}, \ldots, C_{k}$ which are pairwise disjoint and nonhomotopic. If $d\left(C_{i}, C_{j}\right) \geqslant 4$ for all $i, j \in\{1, \ldots, k\}$, then $G$ can be 5 -colored.

Proof. Form $G\left(C_{1}, \ldots, C_{k}\right)$ as defined above. Note that this graph is a triangulation of the sphere and that the induced subgraph on each set $R\left(C_{i}\right)$ and $L\left(C_{i}\right)$ is a cycle. Then $G\left(C_{1}, \ldots, C_{k}\right)$ can be 4-colored [4] from which $G-\left\{C_{1}, \ldots, C_{k}\right\}$ inherits a 4-coloring which we shall extend to a 5-coloring of $G$. Each cycle in $\left\{R\left(C_{i}\right), L\left(C_{i}\right)\right.$ : $i=1, \ldots, k\}$ has been colored with (at most) 3 colors (since, for example, all vertices of $R\left(C_{i}\right)$ are adjacent to $\left.x_{R}^{i}\right)$. Suppose $L\left(C_{i}\right)$ and $R\left(C_{i}\right)$ have received the same triple of colors, say $\{1,2,3\}$. Then colors $\{4,5\}$ can be alternated on $C_{i}$; if $C_{i}$ has odd length, alternate these colors, leaving the vertex of degree 4 to the end at which point it can receive one of the 5 colors. If $L\left(C_{i}\right)$ and $R\left(C_{i}\right)$ have different triples, say $\{1,2,3\}$ and $\{1,2,4\}$, we replace colors $\{3,4\}$ by color 5 on $L\left(C_{i}\right)$ and $R\left(C_{i}\right)$, and use colors $\{3,4\}$ on $C_{i}$ as above. Since $d\left(C_{i}, C_{j}\right) \geqslant 4$, no two vertices from distinct cycles in $\left\{L\left(C_{i}\right), R\left(C_{i}\right): i=1, \ldots, k\right\}$ are adjacent; thus this 5-coloring is proper.

Main results. We now prove our main result. We need to consider $G$ embedded on a surface and simultaneously its representation $G_{k}$ on $P_{k}$; when we alter $G$ or $G_{k}$ we carry out the corresponding alteration on the other.

ProOF OF THEOREM 1. We assume $G$ has a 2-cell embedding on a surface of genus $k \geqslant 1$ and a representation $G_{k}$ on $P_{k}$ with all edges of length less than $\varepsilon=1 / 5$. As in [3] we begin by extending $G$ to an orderly triangulation of the surface.

First we subdivide every nontriangular face by adding a vertex adjacent to all vertices on the face boundary (and add these new vertices and edges to $G_{k}$ ). If any new edge has length $\varepsilon$ or more, we subdivide it by adding new vertices along the edge. We repeat the above process until the resulting graph $G^{\prime}$ is a triangulation with all edges of length less than $\varepsilon$. Finally we create $G^{\prime \prime}$ by erasing all vertices inside a contractible 3- or 4-cycle and subdividing any resulting quadrilaterals. If $G^{\prime \prime}$ can be 
5-colored, then so can $G^{\prime}$ by Lemma 1 . Then $G$ inherits a 5-coloring from $G^{\prime}$ since no edge of $G$ was subdivided.

Thus we assume $G$ is an orderly triangulation and try to 5-color it. For each $i=1, \ldots, k$ we consider the "handle" in the polygon $P_{k}$ with sides labelled $a_{i}, b_{i}$, $a_{i}^{-1}$, and $b_{i}^{-1}$ (see Figure 2); note that one point $S$ is common to all $4 k$ sides of $P_{k}$. Let $p_{i}$ be the set of all points of $P_{k}$ at distance $1 / 2$ from the side $b_{i}$ (see Figure 2). Thus $p_{i}$ is a path from the midpoint of $a_{i}$ to the midpoint of $a_{i}^{-1}$ and represents an nnh-cycle on the original surface (but which is not necessarily a cycle in the graph). Let $L_{i}$ be the set of all points of $P_{k}$ which lie to the left of $p_{i}$, as it is traversed from $a_{i}$ to $a_{i}^{-1}$, and within distance $\varepsilon$ of $p_{i}$.

We claim that within $L_{i}$ there is a path in $G_{k}$, starting and ending at corresponding edges or vertices of $a_{i}$ and $a_{i}^{-1}$, which represents an nnh-cycle in $G$. To find such a path, color a region (or face) of $G_{k}$ blue if it meets the set $L_{i}$ but does not cross $p_{i}$. Since all edges have length less than $\varepsilon$ and $G$ is a 2-cell embedding, one component of the boundary of the blue region lies in $L_{i}$, giving the path in $G_{k}$ and the corresponding nnh-cycle in $G$. Within the latter cycle, find $C_{i}^{\prime}$ which is a minimal nc-cycle.

Let $R\left(C_{i}^{\prime}\right)$ be the set of neighbors of $C_{i}^{\prime}$ which lie to the right of $C_{i}^{\prime}$, as it is traversed from $a_{i}$ to $a_{i}^{-1}$ in $G_{k}$. By Lemma 2 we can find a nice nnh-cycle $C_{i}$ within $C_{i}^{\prime} \cup R\left(C_{i}^{\prime}\right)$, all vertices and edges of which lie within $\varepsilon$ of $p_{i}$ in $P_{k}$.

Clearly $C_{1}, C_{2}, \ldots, C_{k}$ are pairwise disjoint and nonhomotopic. By Lemma $3, G$ is 5-colorable provided $d\left(C_{i}, C_{j}\right)>3$ for all $i, j \in\{1,2, \ldots, k\}$. The shortest path from $C_{i}$ to $C_{j}(i \neq j)$ and the shortest path from $C_{i}$ to $C_{i}$ which induces an nc-cycle $C_{i}^{*}$ lie along a path (in $P_{k}$ ) from $C_{i}$ to $S$ and from (another copy of) $S$ to $C_{j}$ (or $C_{i}$ ). Such a path has (Euclidean) length at least $2(1 / 2-\varepsilon)$. Thus $d\left(C_{i}, C_{j}\right)>(1-2 \varepsilon) / \varepsilon \geqslant 3$ when $\varepsilon \leqslant 1 / 5$.

We can more easily see that short edges ensure 7-colorability.

THEOREM 2. Suppose $G$ has a 2-cell embedding on a surface of genus $k \geqslant 1$ and $a$ representation $G_{k}$ on $P_{k}$ such that every edge of $G$ has length less than $\varepsilon=1 / 2$. Then $G$ can be 7-colored.

Proof. As in the proof of Theorem 1 we may alter $G$ to become a triangulation with all edges of length less than $\varepsilon$; we do not require the graph to be orderly and so do not concern ourselves with separating 3-and 4-cycles. As before we find minimal nnh-cycles $C_{1}^{\prime}, \ldots, C_{k}^{\prime}$ which are pairwise disjoint and nonhomotopic; these cycles need not be nice. The shortest path from $C_{i}^{\prime}$ to $C_{j}^{\prime}$ has (Euclidean) length at least $1 / 2-\varepsilon+1 / 2$. Thus $d\left(C_{i}^{\prime}, C_{j}^{\prime}\right)>(1-\varepsilon) / \varepsilon \geqslant 1$, when $\varepsilon \leqslant 1 / 2$, and no vertex of $C_{i}^{\prime}$ is adjacent to one of $C_{j}^{\prime}$. Removing the cycles $C_{1}^{\prime}, \ldots, C_{k}^{\prime}$ leaves a planar graph which can be 4-colored; at most 3 more colors are needed on the cycles $C_{i}^{\prime}$, and no coloring conflicts occur in this 7-coloring.

The contrapositive of Theorems 1 and 2 is worth noting.

COROLlaRY 2. Let $G$ be a 6- (respectively 8-) chromatic graph. Then in every embedding of $G$ on a surface of genus $k \geqslant 1(2)$ there are edges of length at least $1 / 5$ $(1 / 2)$. 
Presumably there are $k$-colorability results for $k=6$ and $k \geqslant 8$ similar to those of Theorems 1 and 2. It is a bit surprising that the $\varepsilon$ of these results does not depend upon $k$; however, if we had chosen our $4 k$-gon to be regular with sides of length $s(k)$, as for example with a regular polygon with unit radius or unit area, then the same proofs would show that a graph with all edges of length less than $\varepsilon=s(k) / 5$ (or $\varepsilon=s(k) / 2$ ) can be 5-colored (7-colored).

We note that Theorems 1 and 2 can be interpreted to read that a "locally planar" graph embedded on a surface needs "few" colors. Albertson and Stromquist have called an embedded graph locally planar if there is an $i \geqslant 1$ such that the $i$ th neighborhood of every vertex $v$ (i.e. the induced subgraph on $v$ and all vertices at distance at most $i$ from $v$ ) is embedded in a subset of the surface homeomorphic to a subset of the plane. Graphs which satisfy the hypotheses of Theorems 1 or 2 are locally planar since the second (first) neighborhood of each vertex lies in the representation $G_{k}$ with a circle of radius $2 / 5(1 / 2)$ and in $P_{k}$ each noncontractible cycle has (Euclidean) length at least one.

We conclude with two questions. Although the qualitative nature of Theorem 1 may be its main importance, it would be nice to know or to bound the constant $c_{2}$ of Corollary 1. In particular, if the embedding surface is taken to be one with all nc-cycles (of the surface) of length at least one, is there an edge length bound in terms of this unit of measure?

We ask a question which is a variant on one in [3]. In the proof of Theorem 1 (and of Lemma 3 ) the fifth color is used on relatively few vertices, about half of those of the $C_{i}$ 's or of $R\left(C_{i}\right) \cup L\left(C_{i}\right)$. In [8] it is shown that by removing at most $O((\log k) \sqrt{k n})$ vertices of a graph embedded on a surface of genus $k$ with $n$ vertices, a planar graph results. Hence all but $O((\log k) \sqrt{k n})$ vertices can be 4-colored. Are there constants $M(k)$ such that a graph embedded on a surface of genus $k \geqslant 1$ with all edges suitably short can have all but $M(k)$ vertices 4-colored?

ADDENDUM. These same techniques can be applied to nonorientable surfaces to show that graphs embedded on these surfaces with (similarly) short edges also can be five-colored.

ACKNOWLEDGMENTS. I would especially like to thank Michael O. Albertson and Jan Mycielski for their invaluable help on this problem. I would also like to thank James Callahan, Robert Easton, Patricia Sipe and Walter Stromquist for many helpful conversations, and the Mathematics Department of the University of Colorado in Boulder for providing a stimulating environment for a sabbatical.

\section{REFERENCES}

1. M. O. Albertson and J. P. Hutchinson, On the independence ratio of a graph, J. Graph Theory 2 (1978), 1-8.

2. On six-chromatic toroidal graphs, Proc. London Math. Soc. 41 (1980), 533-556.

3. M. O. Albertson and W. R. Stromquist, Locally planar toroidal graphs are 5-colorable, Proc. Amer. Math. Soc. 84 (1982), 449-456.

4. K. Appell and W. Haken, Every planar map is four-colorable, Bull. Amer. Math. Soc. 82 (1976), $711-712$.

5. B. Bollobas, Graph theory, an introductory course, Springer-Verlag, New York, 1979.

6. P. Erdös, Graph theory and probability. II, Canad. J. Math. 13 (1961), 346-352.

7. M. Fréchet and K. Fan, Initiation to combinatorial topology, Prindle, Weber and Schmidt, Boston, Mass., 1967. 
8. J. R. Gilbert, J. P. Hutchinson and R. E. Tarjan, A separator theorem for graphs of hounded genus, J. Algorithms (to appear).

9. B. Grünbaum, Grötzsch's theorem on 3-colorings, Michigan Math. J. 10 (1963), 303-310.

10. J. P. Hutchinson, On coloring maps made from Eulerian graphs, Proc. Fifth British Combinatorial Conf. (Univ. of Aberdeen), 1975, pp. 343-354.

11. E. Kaiser, Färbungssätze für Graphen auf der projektiven Ehene, dem Torus und dem Kleinschen Schlauch, Wiss. Tech. Hochsch. Ilmenau 20 (1974), 47-53.

12. H. V. Kronk, The chromatic number of triangle-free graphs, Graph Theory and Applications (Proc. Conf., Western Michigan Univ., Kalamazoo, Mich., 1972), Lecture Notes in Math., vol. 303, Springer, Berlin, 1972, pp. 179-181.

13. H. V. Kronk and A. T. White, A 4-color theorem for toroidal graphs, Proc. Amer. Math. Soc. 34 (1972), 83-86.

14. J. Mycielski, personal communication.

15. G. Ringel and J. W. T. Youngs, Solution of the Heawood map coloring problem, Proc. Nat. Acad. Sci. U.S.A. 60 (1968), 438-445.

16. G. Springer, Introduction to Riemann surfaces, Chelsea, New York, 1981.

17. W. R. Stromquist, personal communication.

18. A. T. White, Graphs, groups and surfaces, North-Holland, Amsterdam, 1973.

Department of Mathematics, Smith College, Northampton, Massachusetts 01063 